\title{
Bioprospecção das atividades antioxidante, antibacteriana e antibiofilme contra Corynebacterium ulcerans e toxicidade de Stryphnodendron coriaceum Benth
}

\author{
Bioprospecting of antioxidant, antibacterial and antibiofilm activities against Corynebacterium \\ ulcerans and toxicity of Stryphnodendron coriaceum Benth
}

D. C. P. Santos ${ }^{1 *}$; P. D. B.Gomes ${ }^{1}$; D. S. Leite ${ }^{1}$; B. G. V. Nova ${ }^{1}$; M. B. Alves ${ }^{1,2} ;$ P. R. S. Viana ${ }^{1,3}$; J. A. Rodrigues ${ }^{1}$; A. L. Mattos-Guaraldi ${ }^{4}$; P. S. Sabbadini ${ }^{1,2}$; W. C. A. Firmo ${ }^{1,3^{*}}$

${ }^{1}$ Laboratório de Doenças Bacterianas Respiratórias e Sistêmicas, Universidade Ceuma, 65075-120, São Luís-MA, Brasil

${ }^{2}$ Programa de Pós-graduação em Biodiversidade e Biotecnologia pela Rede BIONORTE, Universidade Federal do Maranhão, 65080-805, São Luís-MA, Brasil

${ }^{3}$ Programa de Pós-graduação em Saúde e Ambiente, Universidade Federal do Maranhão, 65080-805, São Luís-MA, Brasil

${ }^{4}$ Laboratório de Difteria e Corinebactérias de Importância Clínica, Universidade do Estado do Rio de Janeiro, 21040900, Rio de Janeiro-RJ, Brasil

*well_firmo@hotmail.com

(Recebido em 15 de junho de 2020; aceito em 29 de outubro de 2020)

Objetivo do estudo foi avaliar as atividades antioxidante, antibacteriana e antibiofilme contra Corynebacterium ulcerans e toxicidade do Extrato Bruto Hidroalcóolico (EBH) de Stryphnodendron coriaceum Benth (barbatimão). As folhas de $S$. coriaceum foram submetidas a maceração com álcool a $70 \%$. Avaliou-se a atividade antioxidante pelos métodos do 1,1-difenil-2-picrilhidrazil (DPPH) e redução do complexo de fosfomolibdênio. A atividade antibacteriana foi medidia por microdiluição (1:2) para as Concentrações Inibitória Mínima (CIM) e Bactericida Mínima (CBM) e a inibição da formação de biofilme em cinco isolados clínicos e um amostra padrão de C. ulcerans. O estudo da toxicidade foi realizado por testes de hemólise, alelopatia e Tenebrio molitor. O EBH apresentou capacidade antioxidantes, capturando $50,7 \%$ do DPPH na concentração de $0,37 \mu \mathrm{g} / \mathrm{mL}$ e reduzindo $21,99 \%$ do fosfomolibdênio. A CIM observada foi de $500 \mu \mathrm{g} / \mathrm{mL}$ para as amostras, exceto a amostra 809, e o EBH não apresentou aspecto bactericida (CBM). A amostra CDC KC 279 diminuiu a formação do biofilme com interferência do EBH, enquanto, a amostra 2649 sofreu aumento (estatisticamente significativa). A concentração eficiente para hemolisar 50\% das hemácias foi de $460,6 \pm 0,03881 \mu \mathrm{g} / \mathrm{mL}$. No teste alelopático, as sementes apresentaram baixa germinação e não houve diminuição na velocidade de crescimento das radículas em contato com o EBH. A taxa média de sobrevivência de T. molitor foi $60 \%$ no grupo da maior concentração, sem significância estatística $(\mathrm{p}=0,752)$ com o controle, essas características sugerem baixa toxicidade. A espécie vegetal em estudo, apresenta propriedades farmacológicas importantes, que pode levar a formulação de produtos terapêuticos, estudos complementares são necessários.

Palavras-chave: corinebactérias, ensaios biológicos, plantas medicinais

The objective of the study was to evaluate the antioxidant, antibacterial and antibiofilm activities against Corynebacterium ulcerans and the toxicity of the Crude Hydroalcoholic Extract (CHE) of Stryphnodendron coriaceum Benth (barbatimão). The leaves of S. coriaceum were subjected to maceration with $70 \%$ alcohol. The antioxidant activity by the methods of 1,1-diphenyl-2-picrylhydrazyl (DPPH) and redution of the phosphomolybdenum were reported. Antibacterial activity was measured by microdilution (1:2) for Minimum Inhibitory Concentration (MIC) and Minimum Bactericidal (MBC) and inhibition of biofilm formation in five clinical strains and a standard C. ulcerans test. The toxicity study was carried out by testis of hemolysis, allelopathy and Tenebrio molitor. EBH has antioxidant capacity, capturing 50.7\% of DPPH at a concentration of $0.37 \mu \mathrm{g} / \mathrm{mL}$ and $21.99 \%$ of phosphomolibdenum. The MIC observed was $500 \mu \mathrm{g} / \mathrm{mL}$ for all strains, except of strains 809, and the CHE does not have a bactericidal aspect (MBC). The CDC KC 279 standard decreased the biofilm formation with CHE interference, while a 2649 strain increased (statistically significant). The efficient concentration for hemolysing $50 \%$ of the red blood cells was 
$460.6 \pm 0.03881 \mu \mathrm{g} / \mathrm{mL}$. In the allelopathic test, as seeds registered low germination and did not decreases the speed of growth of the roots in contact with CHE. The average concentration rate of $T$. molitor was $60 \%$ in the group with the highest concentration, without statistical significance $(p=0.752)$ with the control, these characteristics suggest low toxicity. A plant species under study, has important pharmacological properties, which can lead to therapeutic substances, necessary complementary studies.

Keywords: corinebacteria, biological tests, medicinal plants

\section{INTRODUÇÃO}

A espécie Corynebacterium ulcerans é um isolado comensal da flora mucosa-cutânea de animais domésticos e selvagens [1], sendo primeiramente descrita por Gilbert e Stewart em 1927 [2], por meio de isolados de pacientes com quadros clínicos semelhantes a difteria e diferenciada como espécie distinta do grupo principal Corynebacterium diphteriae por meio de analises moleculares [3]. Trata-se de bacilos Gram-positivos aeróbicos irregulares, catalase e gelatinase-positiva, sem capacidade móvel e de esporulação, que podem produzir variados quadros clínicos relacionados ao tipo e quantidade de toxina produzida [4].

Frequentemente, o gado bovino é apontado como reservatório principal de $C$. ulcerans, sendo associado a casos de mastites bovinas e demonstrando uma possível transmissão por contato próximo com esses animais [5]. Casos de difteria notificados na Europa [6], Japão [7], Estados Unidos da América [8] e, mais recente, Brasil [9, 10] apontam C. ulcerans como agente causador e animais, domésticos ou não, como reservatórios iniciais, reforçando a ideia de transmissão animal-homem e demonstrando a preocupação com a circulação do patógenos fora de áreas rurais $[4,5]$.

A importância clínica de $C$. ulcerans está relacionada com sua capacidade de albergar o fago codificante de toxina homóloga à Toxina Diftérica (TD), responsáveis pelos quadros de difteria clássica [11], além de quadros pulmonares diversos, sinusite necrosante e ulcerações [9]. A preocupação emergente também se deve a capacidade de desenvolver resistência aos agentes antimicrobianos, encontrando-se relatos de resistência à penicilina, eritromicina e clindamicina, usados comumente na terapêutica $[1,12]$.

O conhecimento empírico levou o homem a utilizar vários recursos naturais a seu favor, entre eles, a utilização de plantas medicinais para o tratamento dos mais variados quadros clínicos. A tradição cultural e crença nesses métodos alternativos em muitas regiões, principalmente países em desenvolvimento, ainda representa um dos principais suportes de cuidado e manutenção a saúde [13]. Inúmeros estudos e pesquisas estão sendo realizados visando validar, cientificamente, os conhecimentos populares sobre a ação das plantas medicinais, a fim de se buscar, principalmente, novos fundamentos para tratamentos alternativos e princípios ativos para fármacos, além de minimizar os efeitos colaterais e toxicológicos, buscando um uso mais seguro e confiável [14].

Stryphnodendron coriaceum Benth, popularmente conhecido como barbatimão [15], é uma planta comum de regiões de cerrado e seu uso é relatado no tratamento de diversas doenças, além de atividade antisséptica, antidiarreica, antimicrobianas e anti-hemorrágicas [16], principalmente, associado aos seus constituintes químicos [17].

Considerando-se a magnitude médico global dos relatos de infecções e resistências por $C$. ulcerans, e a carência de estudos com a espécie vegetal $S$. coriaceum, com enfoque em atividade contra bactérias patogênicas, o presente trabalho teve como objetivo avaliar as atividades antioxidante, antibacteriana e antibiofilme contra $C$. ulcerans e toxicidade do extrato bruto hidroalcóolico $(\mathrm{EBH})$ de $S$. coriaceum, favorecendo a validação de conhecimentos da medicina popular.

\section{MATERIAL E MÉTODOS}

\subsection{Coleta e identificação do material vegetal}


A espécie vegetal S. coriaceum, foi coletada no Parque Nacional da Chapada das Mesas, no município de Estreito-MA e foram preparadas exsicatas para a identificação e registro sob o número 01033, pelo Herbário Ático Seabra da Universidade Federal do Maranhão (UFMA).

\subsection{Preparação do extrato bruto hidroalcóolico}

As folhas foram secas por 15 dias em temperature ambiente e pulverizadas para um pó grosso [18], o qual foi misturado (100g) com álcool a $70 \%$ utilizando hidromódulo de 1:10 (m/v) e submetido a processo extrativo por maceração, durante 7 dias, com agitação diária e sob abrigo de luz, para a preparação do EBH. Em seguida, o material foi filtrado e concentrado em evaporador rotativo, a $80^{\circ} \mathrm{C}[19]$.

\subsection{Avaliação antioxidante}

\subsubsection{Determinação pelo sequestro do radical livre 2,2-difenil-1-pecril-hidrazil (DPPH)}

Uma alíquota de $3,5 \mathrm{~mL}$ de solução do DPPH foi misturada com $0,5 \mathrm{~mL}$ de diferentes concentrações do EBH $(0,09-3 \mu \mathrm{g} / \mathrm{mL})$. Após 30 minutos, a absorbância foi medida a $517 \mathrm{~nm}$. O metanol foi utilizado como branco e a solução do DPPH como controle [20]. A percentagem da atividade sequestradora (\% AS) foi calculada pela seguinte equação:

$$
\% \mathrm{AS}=100 \times\left(A_{\text {controle }}-A_{\text {amostra }}\right) / A_{\text {controle }}
$$

onde $A_{\text {controle }}$ é a absorvância do controle (solução com radical DPPH e metanol) e $A_{\text {amostra }}$ é a absorvância do radical na presença do EBH.

\subsubsection{Determinação pela redução do complexo de fosfomolibdênio}

O EBH foi solubilizado com Dimetilsufóxido (DMSO) a $1 \%$ e solução salina $0,9 \%$ e obteve-se uma solução na concentração de $200 \mu \mathrm{g} / \mathrm{mL}$ (concentração padrão para o teste). Retirou-se uma alíquota de $0,4 \mathrm{~mL}$, que foi adicionado a $4 \mathrm{~mL}$ do complexo de fosfomolibdênio. Uma solução constituída a partir de $0,4 \mathrm{~mL}$ de água destilada e $4 \mathrm{~mL}$ do complexo de fosfomolibdênio foi utilizada como branco. A solução de ácido ascórbico na concentração de $200 \mu \mathrm{g} / \mathrm{mL}$ foi utilizada como solução padrão. Os tubos foram incubados por 90 minutos $95{ }^{\circ} \mathrm{C}$. Procederam-se as leituras das absorbâncias em $695 \mathrm{~nm}$ [21]. A percentagem da atividade antioxidante (\%AA) foi calculada pela equação:

$$
\% \mathrm{AA}=\mathrm{A}_{\text {amostra }}-\mathrm{A}_{\text {branco }} / \mathrm{A}_{\text {padrão }}-\mathrm{A}_{\text {branco }} \mathrm{x} 100
$$

onde $\mathrm{A}_{\text {amostra }}$ é a absorbância de cada amostra, $\mathrm{A}_{\text {branco }}$ é a absorbância do branco e $\mathrm{A}_{\text {padrão }}$ é a absorbância do padrão (ácido ascórbico).

\subsection{Avaliação antibacteriana}

\subsubsection{Microrganismos utilizados no estudo}

Todos os isolados (Tabela 1) foram cedidos pelo Laboratório de Difteria e Corinebactérias de Importância Clínica da Universidade do Estado do Rio de Janeiro e estão armazenadas em meio GC-glicerol 20\% no Laboratório de Doenças Bacterianas Respiratórias e Sistêmicas da Universidade do CEUMA. 
Tabela 1: Isolados de Corynebacterium ulcerans utilizadas no estudo e suas características.

\begin{tabular}{ccc}
\hline Isolados & Origem & Toxicidade \\
\hline CDC KC279 & Pneumonia/Humano/EUA & + \\
809 & Pneumonia/Humano/Brasil & + \\
2590 & Difteria/Humano/Brasil & + \\
2625 & Humano/Brasil & + \\
2649 & Cão/Brasil & - \\
2652 & Cão/Brasil & - \\
\hline
\end{tabular}

CDC: Centers for Disease Control and Prevention, Atlanta, GO, USA; EUA: Estados Unidos da América; +: presença do gene que codifica a toxina diftérica (TD); -: ausência do gene que codifica a TD.

\subsubsection{Determinação das concentrações inibitória mínima (CIM) e bactericida mínima (CBM)}

A determinação da concentração inibitória mínima (CIM) foi realizada através de ensaio de microdiluição em placa de 96 poços de poliestireno. Uma alíquota de $10 \mu \mathrm{L}$ de suspensão bacteriana $\left(1,5 \times 10^{8} \mathrm{UFC} / \mathrm{mL}\right)$ foi adicionada a uma diluição seriada (1:2) da solução do EBH (preparada com DMSO a $1 \%$ e solução saliana $0,9 \%$ com obtenção da concentração inicial de $2000 \mu \mathrm{g} / \mathrm{mL}$ ) preparada em Trypticase Soy Broth (TSB) e incubada durante $48 \mathrm{~h}$ a $37^{\circ} \mathrm{C}$. Solução de resazurina $0,03 \%$ foi utilizada como indicador de crescimento [22] e as alterações de cor de roxo para rosa foram registradas como crescimento do microrganismo. A concentração mais baixa na qual não ocorreu alteração de cor foi considerada como CIM. Cloranfenicol $(30 \mu \mathrm{g})$ foi utilizado como controle positivo. Posteriormente, alíquotas dos poços onde não se observou crescimento do microrganismo foram semeadas em Agar Mueller Hinton e incubadas durante $48 \mathrm{~h}$ a $37^{\circ} \mathrm{C}$ para determinar a concentração bactericida mínima $(\mathrm{CBM})$, que correspondeu à concentração mínima do EBH necessária para eliminar os microrganismos [23]. O EBH foi considerado como agente bacteriostático ou bactericida quando se observou razão $\mathrm{CBM} / \mathrm{CIM}>4$ ou razão $\mathrm{CBM} / \mathrm{CIM} \leq 4$, respectivamente [24].

\subsubsection{Ensaio de inibição da formação de biofilme}

O teste foi realizado em placas de microdiluição de 96 poços de poliestireno. As suspensões bacterianas $(200 \mu \mathrm{L}, \mathrm{DO} 0,2 ; 570 \mathrm{~nm})$ foram adicionadas aos poços contendo ou não a CIM do $\mathrm{EBH}(100 \mu \mathrm{L})$. No primeiro ensaio, a suspensão bacteriana e o EBH foram adicionados ao mesmo tempo aos poços da microplaca, que foi incubada por $48 \mathrm{~h}$ a $37^{\circ} \mathrm{C}$. O conteúdo de cada poço foi aspirado e lavado com tampão fosfato-alcalino (Phosphate-bufferid saline [PBS] pH 7,2; 0,01M) e as células bacterianas remanescentes foram fixadas com $99 \%$ de metanol e coradas com $2 \%$ de cristal violeta. $\mathrm{O}$ corante ligado foi então solubilizado com ácido acético glacial a 33\% e a solução foi medida em 550nm [25].

\subsection{Avaliação da toxicidade in vitro e in vivo}

\subsubsection{Avaliação hemolítica}

O estudo foi aprovado pelo comitê de ética em pesquisa com seres humanos da Universidade Ceuma com o número do parecer 1.732.522. Para o ensaio, foram obtidas hemácias de humano voluntário saudável e sem histórico de uso de drogas 3 semanas antes da coleta da amostra.

O ensaio foi realizado segundo o método de Yang, Sun e Fang (2005) [26]. Preparou-se uma suspensão de hemácias a $1 \%$ e $0,5 \mathrm{~mL}$ desta suspensão foi misturada a $0,5 \mathrm{~mL}$ de soluções do $\mathrm{EBH}$ $(1000$ a $50 \mu \mathrm{g} / \mathrm{mL})$, com posterior incubação por $1 \mathrm{~h}$ a $37^{\circ} \mathrm{C}$. As soluções foram, então, centrifugadas a $3000 \mathrm{rpm}$ por $5 \mathrm{~min}$. A absorbância do sobrenadante foi medida a $540 \mathrm{~nm}$. As suspensões de hemácias acrescidas de solução salina e de água destilada foram, respectivamente, os controles hemolíticos mínimo e máximo. 


\subsubsection{Atividade alelopática}

O teste do potencial alelopático do EBH foi feito a partir de diferentes concentrações (5-20\%), tendo como controle negativo água destilada. Sementes de alface foram distribuídas em grupos de 20 em placas de Petri forradas com discos de papel de germinação embebecido com $3 \mathrm{~mL}$ das concentrações do EBH. Logo após, foram colocadas para germinação em temperatura ambiente e fotoperíodo de 12 horas. As variáveis analisadas tratam-se da porcentagem de germinação de sementes e o índice de velocidade de crescimento de raiz (IVCR). Após a protrusão da raiz, as placas foram fotografadas, por quatro dias, e analisadas em software Image J [27].

\subsubsection{Bioensaio utilizando larvas de Tenebrio molitor}

Larvas de T. molitor foram distribuídas placas de Petri em dois grupos (teste e controle) contendo 10 larvas cada. Injetou-se $10 \mu \mathrm{L}$ na região caudal das larvas do EBH $(1000$ a $50 \mu \mathrm{g} / \mathrm{mL})$. As larvas permaneceram em temperatura ambiente e a taxa de sobrevivência foi observada em intervalos de 24 h, durante 5 dias. Para estabelecer a morte das larvas, verificou-se visualmente a melanização e a resposta aos estímulos físicos. O controle negativo foi DMSO a $1 \%$ e solução salina $(0,9 \%)$ [28].

\subsection{Análise estatística}

Utilizou o programa GraphPad Prism 6 para análises dos dados, sendo esses expressos como média \pm desvio-padrão. Para o teste que determina s concentração eficiente de ação hemolítica utilizou-se regressão não linear. Nos ensaios que verificaram as diferenças entre os grupos, a comparação foi realizada com o teste de Tukey. A curva de sobrevivência no ensaio com as larvas de T. molitor foi realizada com os testes de Kaplan-Meier, analisando-se os resultados com o teste Log-Rank (Mantel-Cox). Considerou-se $\mathrm{p}<0,05$ como significativo. Os testes foram realizados uma única vez em triplicata.

\section{RESULTADOS}

\subsection{Capacidade antioxidante}

Observou-se o perfil antioxidante do extrato de S. coriaceum por duas técnicas distintas. Para o método de inibição do complexo de fosfomolibdênio encontrou-se uma média de porcentagem de $21,99 \%$, sendo uma redução media $63 \%$ para o controle (ácido ascórbico). Para a redução de radicais livres do DPPH observou-se uma ação média de captura de radicais de 50,70\% para a concentração $0,37 \mu \mathrm{g} / \mathrm{mL}$; enquanto a solução-controle do DPPH apresentou uma captura de $67,30 \%$ para a mesma concentração.

\subsection{Atividade antibacteriana e antibiofilme contra Corynebacterium ulcerans}

O estudo da atividade antibacteriana pelo teste de microdiluição, determinou a CIM de 500 $\mu \mathrm{g} / \mathrm{mL}$ para quase todas as cepas, exceto para o isolado 809. Contudo, não foi possível determinar a CBM pelas concentrações testadas, ou seja, não houve concetração do extrato em que se observou ação bactericida minima. Assim, não se pode verificar a relação da CBM/CIM, que determina a capacidade da espécie vegetal de ter efeito bacteriostático ou bactericida (Tabela 2). 
Tabela 2: Concentração inibitória e bactericida mínima do extrato bruto hidroalcóolico de Straphynodendron coriaceum contra Corynebacterium ulcerans.

\begin{tabular}{cccc}
\hline & \multicolumn{3}{c}{ Stryphnodendron coriaceum } \\
\cline { 2 - 4 } Corynebacterium ulcerans & CIM & CBM & CBM/CIM \\
\hline CDC KC279 (tox + ) & 500 & ND & ND \\
809 (tox + ) & ND & ND & ND \\
$2590($ tox +$)$ & 500 & ND & ND \\
$2625($ tox +$)$ & 500 & ND & ND \\
$2649($ tox -$)$ & 500 & ND & ND \\
2652 (tox -$)$ & 500 & ND & ND
\end{tabular}

CDC: Centers for Disease Control and Prevention, Atlanta, GO, USA; tox +: presença do gene da toxina diftérica; tox -: ausência do gene da toxina diftérica; CIM: Concentração inibitória mínima; CBM:

Concentração bactericida mínima; ND: Não foi possível definir com as concentrações avaliadas. O controle

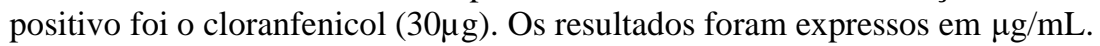

Em relação a formação e inibição do biofilme pelo EBH, notou-se que todas as cepas, são produtoras de biofilme em poliestireno com intensidades variadas e independente ou não de produzir TD. Observa-se que a amostra KC 279 diminuiu com a presença do EBH, enquanto as amostras 809, 2649 e 2652 aumentaram a formação do biofilme, contudo, somente a amostra 2649 apresentou diferença estatisticamente significativa para o aumento $(\mathrm{p}<0,05)$ (Figura 1).

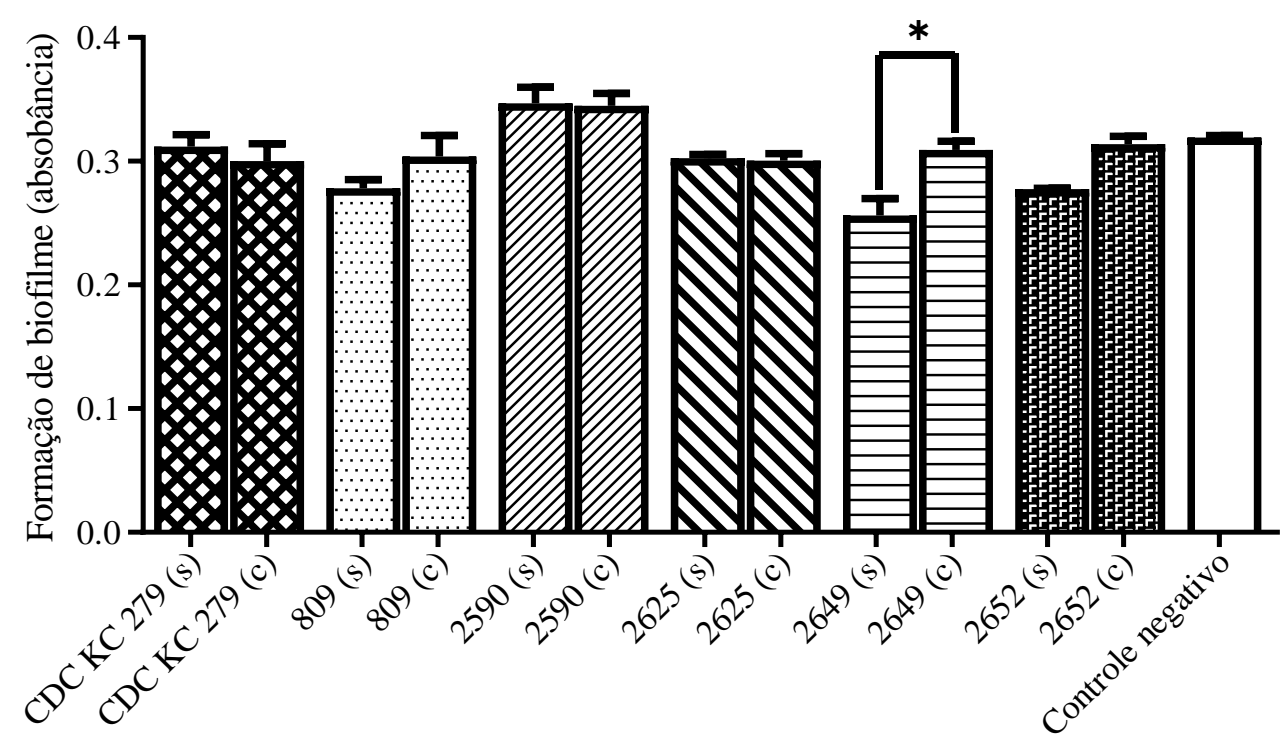

Figura 1: Formação de biofilme por Corynebacterium ulcerans com e sem interferência do extrato bruto hidroalcoólico de Straphynodendron coriaceum. CDC: Centers for Disease Control and Prevention, Atlanta, GO, USA; C: Crescimento do isolado com interferência do EBH; S: Crescimento do isolado sem interferência do EBH; *As diferenças foram consideradas significativas quando $p<0,05$ (Teste de Tukey).

\subsection{Toxicidade da espécie vegetal}

Em relação a toxicidade sobre hemácias, a concentração eficiente para hemolisar $50 \%$ das hemácias $\left(\mathrm{CE}_{50}\right)$ foi de $460,6 \pm 0,03881 \mu \mathrm{g} / \mathrm{mL}$.

No ensaio alelopático, as sementes apresentaram baixa germinação em contato com o EBH de S. coriaceum e não houve diminuição da velocidade do crescimento das radículas e das partes aéreas nas concentrações testadas em comparação ao controle (Tabela 3). 
Tabela 3: Efeito alelopático do extrato bruto hidroalcóolico de Straphynodendron coriaceum sobre sementes de alface.

\begin{tabular}{ccc}
\hline Concentração do extrato $(\%)$ & $\begin{array}{c}\text { Porcentagem de } \\
\text { germinação }\end{array}$ & $\begin{array}{c}\text { Comprimento da } \\
\text { radícula }\end{array}$ \\
\hline 0 & $76,7^{\mathrm{a}}$ & $2,9^{\mathrm{a}}$ \\
5 & $50^{\mathrm{b}}$ & $2,5^{\mathrm{a}}$ \\
10 & $60^{\mathrm{b}}$ & $3,4^{\mathrm{a}}$ \\
15 & $36,7^{\mathrm{c}}$ & $3,3^{\mathrm{a}}$ \\
20 & $20^{\mathrm{c}}$ & $3,0^{\mathrm{a}}$ \\
\hline
\end{tabular}

Médias seguidas por letras iguais na coluna não diferem estatisticamente entre si pelo Teste de Tukey $(<0,05)$.

Os ensaios in vivo com as larvas de T. molitor demonstraram que a média de sobrevivência foi $60 \%$ no grupo da maior concentração $(1000 \mu \mathrm{g} / \mathrm{mL})$, enquanto apresentou taxa de sobrevivência de $100 \%$ em relação às outras concentrações, sem significância estatística $(\mathrm{p}=0,752)$ quando comparadas com o controle, sugerindo baixa toxicidade (Figura 2).

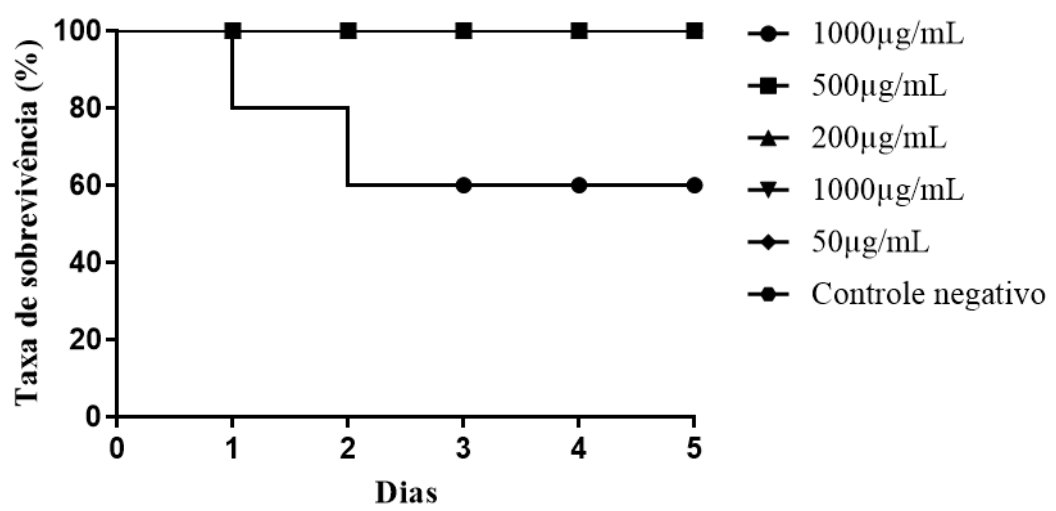

Figura 2: Taxa de sobrevivência de larvas de Tenebrio molitor após injeção com extrato bruto hidroalcóolico de Straphynodendron coriaceum. As diferenças foram consideradas significativas quando $p<0,05$ (Teste Kaplan-Meier e Teste Log-Rank [Mantel-Cox]).

\section{DISCUSSÃO}

O metabolismo secundário vegetal é responsável pela produção de diversos compostos orgânicos (nitrogenados, fenólicos, terpenos, alcaloides, entre outros), que, por sua vez, possuem atividade biológica e são foco de pesquisas de bioativos farmacológicos [29]. Assim, é fácil associar o estudo da bioprospecção de plantas medicinais como tratamento alternativo com a análise preliminar dos seus diferentes constituintes químicos [30].

Firmo (2018) [31] aponta que a possível ação de um espécime vegetal está intimamente relacionada com a concentração de seus compostos químicos. Visto isso, é importante ressaltar que alguns fatores podem influenciar e alterar a taxa de produção desses metabólitos secundários, como sazonalidade, índice pluviométrico, temperatura e altitude, condições de processamento, entre outros. Portanto, a formação de metabolitos secundários é dependente da interação planta-ambiente, ou seja, sua síntese é influenciada e afetada pelas condições ambientais [32].

Silva, Miranda e Conceição (2010) [33] demonstram a presença de esteroides, alcaloides, flavonoides, taninos e saponina em estudos químicos realizados com amostras de folha e casca de S. coriaceum, coletadas no município de Caxias-MA. Firmo (2018) [31] corrobora com esse achado apresentando um perfil fitoquímico para $S$. coriaceum com fenois, taninos, flavanoides, esteroides, saponinas e cumarinas, nessa e outros espécimes do estudo.

A ação antioxidante traz grandes benefícios para a saúde e qualidade de vida, uma vez que protegem o organismo dos danos causados pela formação de radicais livres [34]. A presença de 
flavonoides, segundo Nascimento et al. (2011) [35], está correlacionada a atividade antioxidante de um composto vegetal. Campos e Frasson (2011) [36] afirmam que quanto menor a quantidade de fenois, menos intenso o potencial antioxidante. Oliveira et al. (2011) [37] afirmam que taninos e flavonoides estão entre os compostos metabólicos mais importantes devido sua ligação direta com atividade antioxidante. Esses resultados comparados com estudos que demonstram a presença desses compostos na espécie vegetal avaliada podem explicar sua ação antioxidante encontrada nessa pesquisa.

Costa et al. (2012) [38] demonstram a capacidade antioxidante pelo método de DPPH de um extrato aquoso das folhas de uma espécie do gênero Stryphnodendron, encontrando uma concentração eficiente de 46,3 $\pm 12,3 \mu \mathrm{g} / \mathrm{mL}$ (controle 52,8 $\mu \mathrm{g} / \mathrm{mL} ; \mathrm{p}<0,001$ ). Zocoler et al. (2009) [39], apontaram não haver atividade antioxidante por $S$. obovatum, usando o método inibição do complexo de fosfomolibdênio, com diferença significaiva $(\mathrm{p}<0,005)$ entre a ação do extrato bruto (5,39\%) e ácido ascórbico (100\%). Santos-Filho, Ferreira e Gouvêa (2011) [40], no entanto, determinaram o alto potencial antioxidante de $S$. adstringens em teste in vitro, atingindo a capacidade máxima com uma concentração de $62 \pm 0,91 \mu \mathrm{g} / \mathrm{mL}$, além de avaliarem os altos teores de compostos fenólicos, flavonoides e proantocianidina, demonstrando potencial anticancerígeno e reafirmando a influência da concentração dos compostos vegetais.

Não é possível fazer uma comparação de resultados pelos diferentes métodos aplicados, porém o extrato em estudo, assim como outros espécimes do gênero Sryphnodendron, apresentaram melhores resultados frente a redução do radical de DPPH.

Em relação a metodologia aplicada para avaliar a CIM do extrato, Bona et al. (2014) [41] justificam que o teste de microdiluição em placa permite uma economia de espaço, meios de cultura e reagente, além de possibilitar a determinação quantitativa da CIM, mostrando melhor desempenho e sensibilidade que outros testes, como difusão em poços. Assim, pela microdiluição, observou-se ação moderada do extrato $(\mathrm{CIM}=500 \mu \mathrm{g} / \mathrm{mL})$ contra as corineas, ainda que o gênero Stryphnodendron apresente características de possível ação antimicrobiana contra outros gêneros relatados na literatura.

O único estudo que trabalha laboratorialmente a ação de $S$. coriaceum contra espécies de corinebactérias é de Firmo (2018) [31], o qual demonstra baixa ou moderada ação antimicrobiana contra cepas produtoras ou não de toxina diftérica, corroborando com os resultados da avaliação antimicrobiana desse estudo. Firmo (2018) [31] demontra uma concetração de com $1.160 \mu \mathrm{g} / \mathrm{mL}$ para CIM contra uma cepa de C. ulcerans, sem ação bactericida. Essa mesma CIM se repete para cepas de $C$. diphtheriae e $C$. pseudodiphtheriticum, com variados resultados para CBM, sendo o $S$. coriaceum caracterizado somente como bacteriostático (CIM/CBM >4), semelhante ao estudo.

No entanto, estudos feitos por Souza et al. (2013) [42] apontam uma espécime de Stryphnodendron como uma das plantas medicinais mais usadas em tratamentos de quadros infeciosos, demonstrando sua possível ação antimicrobiana, concordando com Bessa et al. (2013) [43]. Conceição et al. (2011) [16] corroboram nesse fato apontando o uso de S. coriaceum em infecções do útero, e Soares et al. (2008) [44] relatam ação do gênero Stryphnodendon com a atividade antibacteriana contra cepas de Streptococcus mitis e Lactobacillus casei, com uma CIM de $350 \mu \mathrm{g} / \mathrm{mL}$ para ambas as cepas.

A resistência bacteriana aos antibióticos é atualmente um dos problemas de saúde médico global mais relevantes, uma vez que muitas bactérias, anteriormente suscetíveis, deixaram de reagir a ação desses agentes [45]. Diversos estudos comprovam um aumento na taxa de resistência aos antibióticos por espécies de corinebactérias, principalmente aos $\beta$-lactâmicos, clindamicina, eritromicina, ciprofloxacino e gentamicina, usados no tratamento dessas infecções $[1,46]$. Mina et al. (2011) [47] relataram o primeiro caso de multirresistência em $C$. diphtheriae, no qual o isolado apresentou resistência a eritromicina, clindamicina, trimetoprim-sulfametoxazol e tetraciclina.

Plantas medicinais, atualmente, são alvo de pesquisas para avaliação de seu potencial farmacológico. Desoti et al. (2011) [48] confirmam a possibilidade de usar essas plantas para o desenvolvimento de novos antimicrobianos, tanto para ação individual, quanto associação com outros medicamentos já utilizados na terapêutica. Firmo (2018) [31] demonstra que, mesmo não apresentando efeito antibacteriano satisfatório, S. coriaceum desempenha uma ação eficiente quando exposta a administração conjuntas com outras drogas antimicrobianas. 
A formação de biofilme é um aglomerado celular organizado de bactérias, em uma superfície biótica ou abiótica, que produzem substâncias extracelulares matricial para proteção conjunta, aumentando a resistência e tonando sua configuração uma preocupação no meio médico hospitalar [49]. É crescente o interesse em pesquisar produtos naturais que possuam ação inibitória na formação de biofilme por diversos micro-organismos [50, 51].

Assim como avaliado no estudo, Gomes et al. (2013) [25] encontraram a capacidade de isolados de corinebatéria em melhorar a formação de biofilme em uma superfície de vidro ao serem expostos a subconcentrações de eritromicina e penicilina. Estudos apontam a ligação ao fibrinogênio, fibronecina e colágeno como um fator não específico de adesão por cepas de $C$. diphtheriea e $C$. ulcerans [52, 53], assim como pili adesivos como fotor específico [54]. Recentemente, Peixoto et al. (2017) [55] caracterizaram, por analises de bioinformática, a proteína DIP2093, a qual possui similaridade estrutural com a proteína SdrD de Staphylococcus aureus, importante para a formação de biofilme. Além disso, a sequência DIP2093 se mostra altamente conservada entre muitas linhagens de C. ulcerans, C. diphtheriae e C. pseudotuberculosis.

Firmo (2018) [31] realizou um estudo sobre a ação de diversas espécies vegetais contra a formação de biofilme e a erradicação de biofilme já formado de diversas espécies de Corybacterium, entre elas, uma amostra padrão de C. ulcerans (CDC KC279). Nesse estudo o EBH de $S$. coriaceum foi capaz de inibir a formação e de erradicar o biofilme formado por C. ulcerans, com apenas $1 / 4$ da CIM encontrada anteriormente no estudo sobre o espécime. Ainda que haja falta de outros relatos sobre a ação inibitória de $S$. coriaceum na formação de biofilme bacteriano, Freitas et al. (2018) [56] e Luiz et al. (2015) [57] demonstraram o efeito antibiofilme de espécie do gênero de Stryphnodendron sobre isolados de Candida albicans e Candida glabrata. O extrato de Sryphnodendon barbatiman demonstrou redução de $95 \%$ da formação de biofilme por Fusobacterium nucleatum em concentração de $100 \mathrm{mg} / \mathrm{mL}$, sendo estasticamente semelhante ao controle com clorexidina a $0,12 \%$ (98\% de inibição) [58].

Plantas medicinais apresentam uma variedade de compostos metabólitos primários e secundários, que podem exercer tanto efeito benéficos quanto maléficos sobre o organismo, necessitando de teste toxicológicos para avaliação de seu risco/benefício [31]. De acordo com Kumar et al. (2009) [59], a citotoxicidade é diretamente proporcional à concentração da amostra e ao tempo de exposição. Firmo (2018) [31] corrobora que a toxidade está relacionada com a concentração dos compostos químicos do extrato.

A ocorrência de hemólise após a exposição ao produto de teste pode ser diretamente correlacionada com a sua citotoxicidade e utilizada como o primeiro passo na triagem toxicológica in vitro, por ser possível se obter dados significativos com testes simples [60]. O uso de modelos invertebrados também apresenta vantagens em relação os modelos convencionais de mamíferos, incluindo custos mais baixos, resultados mais rápidos e menos questões éticas [61].

Firmo (2018) [31] avaliou a ação hemolítica de diversos espécimes vegetais, dentre as quais $S$. coriaceum apresentou a maior $\mathrm{CE}_{50}(173,60 \pm 0,03922 \mu \mathrm{g} / \mathrm{mL})$, considerada a espécime com maior capacidade de hemólise. Baldivia et al. (2018) [62] avaliaram a atividade hemolítica de uma espécie do gênero Stryphnodendron, no qual se observou não só a ausência de atividade hemolítica pelo extrato nas concentrações de 50-125 $\mu \mathrm{g} / \mathrm{mL}$, como a proteção contra hemólise oxidativa quando se expôs os eritrócitos a um agente oxidante, apresentando melhor atividade protetiva que o controle com ácido ascórbico.

Os taninos apresentam a capacidade de interagirem com proteínas e outras macromoléculas, conferindo-o atividade tóxica e aglutinante [43]. Além disso, Bukowska e Kowalska (2004) [63] afirmam que taninos e fenóis são capazes de promover ação hemolítica por oxidação da hemoglobina. As saponinas são compostos capazes de se ligarem e desorganizarem a membrana plasmática das hemácias, levando a uma hemólise por aumento da permeabilidade [43].

Segundo Firmo (2018) [31], S. coriaceum causa uma mortalidade significativa $(p=0,0414)$ na concentração de $1000 \mu \mathrm{g} / \mathrm{mL}$, quando comparado com o controle, sugerindo o EBH nessa concentração é tóxico para T. molitor, o que corrobora com os resultados encontrados no presente estudo de que a espécie vegetal não apresenta toxicidade nesse modelo in vivo.

A presença de taninos em $S$. coriaceum sugere toxicidade, também, pela facilidade em quelar íons metálicos, importantes cofatores enzimáticos para redução digestiva, com efeito ligado diretamente à sua concentração [64, 65]. Essa ligação reduz o crescimento e sobrevivência de 
artrópodes por inativação de enzimas digestiva, deficiência de absorção nutricional e lesão tecidual, embora outro estudo aponta a diferença de resultados em diferentes formas evolutivas do inseto $[64,66]$.

Muitas plantas medicinais apresentam efeito alelopático, ou seja, o efeito benéfico ou danoso que uma planta exerce sobre outra, por meio da produção de compostos químicos liberados no ambiente, sendo avaliada como alternativa ao uso de herbicidas, inseticidas e nematicidas [67]. Sobre essa ação alelopática, estudos feitos com amostras do gênero Stryphnodendron corroboram com os resultados encontrados nesse estudo, apresentando protencial alelopático por inibição da germinação em alface com $63,11 \%$, utilizando o extrato aquoso das folhas [68] e inibição de $65 \%$ e $35 \%$ com os extratos metanólico e clorofórmio de pição-preto, respectivamente [69].

\section{CONCLUSÃO}

O extrato de $S$. coriaceum apresentou moderada atividade antimicrobiana contra isolados de $C$. ulcerans, além de não apresentar efeito na inibição da formação de biofilme por essa espécie, demonstrando sua baixa viabilidade para a formulação de antimicrobianos contra essa espécie, porém sem descartar a possibilidade de ação contra espécies de outros gêneros. Sua baixa toxicidade e sua boa capacidade antioxidante demonstranda pelo teste do DPPH são indicativos de seu potencial para estimular e direcionar o desenvolvimento de novos fármacos e métodos alternativos para o tratamento e manutenção de diversos quadros de saúde não infeciosos.

\section{REFERÊNCIAS BIBLIOGRÁFICAS}

1. Hacker E, Antunes CA, Mattos-Guaraldi AL, Burkovski A, Tauch A. Corynebacterium ulcerans, an emerging human pathogen. Future Microbiol. 2016 Aug;11(9):1191-208, doi: 10.2217/fmb-2016-0085.

2. Gilbert R, Stewart FC. Corynebacterium ulcerans: a pathogenic microorganism resembling $C$. diphtheriae. J Lab Clin Med. 1927;12:756-61.

3. Torres LFC, Ribeiro D, Hirata Junior R, Pacheco LGC, Souza MC, Santos LS, Santos CS, Salah M, Costa MM, Ribeiro MG, Selim SA, Azevedo VAC, Mattos-Guaraldi AL. Multiplex PCR for identification and toxigenicity of Corynebacterium group of zoonotic potential and an overview of human and animal infections. Mem Inst Oswaldo Cruz. 2013;108(3):272-9.

4. Dias AASO, Santos LS, Sabbadini PS, Santos CS, Silva Junior FC, Napoleão F, Nagao PE, Villas-Bôas MHS, Hirata Junior R, Mattos-Guaraldi AL. Difteria pelo Corynebacterium ulcerans: uma zoonose emergente no Brasil e no mundo. Rev Saúde Publ. 2011;45(6):1176-91.

5. Mattos-Guaraldi AL, Hirata R, Azevedo VAC. Corynebacterium diphtheriae, Corynebacterium ulcerans and Corynebacterium pseudotuberculosis-general aspects. In: Burkovski A, editor. Corynebacterium diphtheriae and Related Toxigenic Species. Dordrecht: Springer; 2014. p. 15-37.

6. Leggett BA, Zoysa A, Abbott YE, Leonard N, Markey B, Efstratiou A. Toxigenic Corynebacterium diphtheriae isolated from a wound in a horse. Vet Rec. 2010;166:656-7, doi: 10.1136/vr.b4846.

7. Katsukawa C, Kawahara R, Inoue K, Ishii A, Yamagishi H, Kida K, Nishino S, Nagahama S, Komiya T, Iwaki M, Takahashi M. Toxigenic Corynebacterium ulcerans isolated from the domestic dog for the first time in Japan. Jpn J Infect Dis. 2009 Jan;62(2):171-2.

8. Hall AJ, Cassiday PK, Bernard KA, Bolt F, Steigerwalt AG, Bixler D, Pawloski LC, Whitney AM, Iwaki M, Baldwin A, Dowson CG, Komiya T, Takahashi M, Hinrikson HP, Tondella ML. Novel Corynebacterium diphtheriae in domestic cats. Emerg Infec Dis. 2010 Apr;16(4):688-9, doi: 10.3201/eid1604.091107.

9. Mattos-Guaraldi AL, Sampaio JLM, Santos CS, Pimenta FP, Pereira GA, Pacheco LGC, Miyoshi A, Azevedo V, Moreira LO, Gutierrez, Costa JLF, Costa-Filho R, Damasco PV, Camello TCF, Hidrata Junior R. First detection of Corynebacterium ulcerans producing a diphtheria-like toxin in a case of human with pulmonary infection in the Rio de Janeiro metropolitan area, Brazil. Mem Inst Oswaldo Cruz. 2008;103(4):396-400, doi: 10.1016/S0723-2020(87)80060-7.

10. Simpson-Lourêdo L, Silva CMF, Hacker E, Souza NF, Santana MM, Antunes CA, Nagao PE, Hirata Junior R, Burkovski A, Villas-Bôas MHS, Mattos-Guaraldi AL. Detection and virulence potential of a phospholipase D-negative Corynebacterium ulcerans from a concurrent diphtheria and infectious mononucleosis case. Antonie van Leeuwenhoek. 2019 Feb;112(7):1055-65, doi: 10.1007/s10482-01901240-4. 
11. Burkovski A. Diphtheria and its etiological agents. In: Burkovski A, editor. Corynebacterium diphtheriae and related toxigenic species. Dordrecht: Springer; 2014. p. 1-14, doi: 10.1007/978-94-007-7624-1_1.

12. Katsukawa C, Komiya T, Yamagishi H, Ishii A, Nishino S, Nagahama S, Iwaki M, Yamamoto A, Takahashi M. Prevalence of Corynebacterium ulcerans in dogs in Osaka, Japan. J Med Microbiol. 2012 Feb;61(2):266-73, doi: 10.1099/jmm.0.034868-0.

13. Brasil. Ministério da Saúde. Secretaria de Ciência, Tecnologia e Insumos Estratégicos. Departamento de Assistência Farmacêutica e Insumos Estratégicos. A fitoterapia no SUS e o programa de pesquisas de plantas medicinais da central de medicamentos. Brasília (DF): Ministério da Saúde; 2006.

14. Firmo WCA, Menezes VJM, Possos CEC, Dias CN, Alves LPL, Dias ICL, Santos Neto M, Olea RSG. Contexto histórico, uso popular e concepção científica sobre plantas medicinais. Cad Pesq. 2011 Dec;18(especial):90-95.

15. Lemos ICS, Lacerda GM, Dalmondes GA, Barbosa R, Fernandes GP, Kerntopf MR. Recursos naturais para tratamento de anemia em crianças: contribuições à medicina tradicional. Rev Saúde Desenv. 2017;11(6):6-21

16. Conceição GM, Ruggieri AC, Araujo MFV, Conceição TTMM, Conceição MAMM. Plantas do cerrado: comercialização, uso e indicação terapêutica fornecida pelos raizeiros e vendedores, Teresina, Piauí. Sci Plen. 2011;7(12):1-6.

17. Lima AK, Amorim ELC. Estudo químico de plantas do nordeste brasileiro visando à obtenção de produtos naturais de potencial atividade biológica. In: Anais do VI Congresso de Iniciação Científica da UFPE; 1998; Recife, PE. Anais eletrônicos: c1998.

18. Farmacopéia Brasileira. 4. ed. Parte II/2º fascículo. São Paulo: Atheneu; 2000.

19. Firmo WCA, Miranda MV, Coutinho GSL, Silveira LMS, Olea RSG. Estudo fitoquímico e avaliação da atividade antibacteriana de Lafoensia pacari (Lythraceae). Publ UEPG Ci Biol Saúde. 2014;20(1):7-12, doi: 10.5212/Publ.Biologicas.v20i1.0001.

20. Firmo WCA, Miranda MV, Coutinho GSL, Barboza JR, Alves LPL, Olea RSG. Determinação de compostos fenólicos e avaliação da atividade antioxidante de Lafoensia pacari (Lythracea). Rev Eletrônica Farma. 2015;12(1):1-10, doi: 10.5216/ref.v12i1.24645.

21. Prieto P, Pineda M, Aguilar M. Spectrophotometric quantitation of antioxidant capacity through the formation of a phosphomolybdenum complex: specific application to the determination of vitamin $\mathrm{E}$. Anal Biochem. 1999;269:337-41.

22. Duarte M, Giordani RB, Carli GA, Zuanazzi JA, Macedo AJ, Tasca, T. A quantitative resazurin assay to determinate the viability of Trichomonas vaginalis and the cytotoxicity of organic solvents and surfactant agents. Exp Parasitol. 2009;123(2):195-8, doi: 10.1016/j.exppara.2009.07.002.

23. Santurio JM, Santurio DF, Pozzatti P, Moraes C, Franchin PR, Alves SH. Atividade antimicrobiana dos óleos essenciais de orégano, tomilho e canela frente a sorovares e Salmonella enterica de origem avícola. Ciênc Rural. 2007 Jun;37(3):803-8, doi: 10.1590/S0103-84782007000300031.

24. Gatsing D, Mbah JA, Garba IH, Tane P, Djemgou P, Nji-Nkah BF. An antisalmonellal agent from the leaves of Glossocalyx brevipes Benth (Monimiaceae). Pak J Biol Sci. 2006;9:84-7, doi: 10.3923/pjbs.2006.84.87.

25. Gomes DLR, Peixoto RS, Barbosa EAB, Napoleão F, Sabbadini PS, Santos KRN, Mattos-Guaraldi AL, Hirata Junior R. SubMICs of penicillin and erythromycin enhance biofilm formation and hydrophobicity of Corynebacterium diphthriae strains. J Med Microbiol. 2013 May;62(5):754-60, doi: 10.1099/jmm.0.052373-0.

26. Yang ZG, Sun HX, Fang WH. Haemolytic activities and adjuvant effect of Astragalus membranaceus saponins (AMS) on the immune responses to ovalbumin in mice. Vaccine. 2005 Oct;23(44):5196-5203, doi: 10.1016/j.vaccine.2005.06.016.

27. Amâncio BCS. Bioatividade de extratos de folhas e frutos de Byrsomina spp. (Malphigiaceae) em Lactuca sativa L [dissertação]. Alfenas (MG): Universidade Federal de Alfenas; 2016. 67 p.

28. Souza PC, Morey AT, Castanheira GM, Bocate KP, Panagio LA, Ito FA, Furlaneto MC, Yamada-Ogatta SF, Costa IN, Mora-Montes HM, Almeida RS. Tenebrio molitor (Coleoptera: Tenebrionidae) as an alternative host to study fungal infections. J Microbiol Methods. 2015;118:182-6, doi: 10.1016/j.mimet.2015.10.004.

29. Peixoto Sobrinho TJS, Castro VTNA, Saraiva AM, Almeida DM, Tavares EA, Amorim ELC. Phenolic content and antioxidant capacity of four Cnidoscolus species (Euphorbiaceae) used as ethnopharmacologicals in Caatinga, Brazil. Afr J Pharm Pharmacol. 2011 Nov;5(20):2310-6, doi: 10.5897/AJPP11.608.

30. Lima Neto GA, Kaffashi S, Luiz WT, Ferreira WR, Silva YSAD, Pazin GV, Violante IMP. Quantificação de metabólitos secundários e avaliação da atividade antimicrobiana e antioxidante de algumas plantas selecionadas do Cerrado de Mato Grosso. Rev Bras Plantas Med. 2015;17(4):1069-77, doi: 10.1590/1983084X/14_161. 
31. Firmo WCA. Bioprospecção de plantas medicinais do cerrado maranhense com propriedades antioxidante, antibacteriana contra Corynebacterium spp e toxicidade: aporte para o desenvolvimento de novos medicamentos [dissertação]. São Luís (MA): Universidade Federal do Maranhão; 2018. 165 f.

32. Gobbo-Neto L, Lopes NP. Plantas medicinais: fatores de influência no conteúdo de metabólitos secundários. Quím Nova. 2007;30(2):374-81, doi: 10.1590/S0100-40422007000200026.

33. Silva NLA, Miranda FAA, Conceição GM. Triagem fitoquímica de plantas de Cerrado, da área de proteção ambiental municipal do Inhamum, Caxias, Maranhão. Sci Plena. 2010;6(2):1-17.

34. Oliveira GLS. Determinação da capacidade antioxidante de produtos naturais in vitro pelo método do DPPH•: estudo de revisão. Rev Bras Plantas Med. 2015 Mar;17(1):36-44, doi: 10.1590/1983084X/12_165.

35. Nascimento JC, Lage LFO, Camargos CRD, Amaral JC, Costa LM, Sousa AN, Oliveira FQ. Determinação da atividade antioxidante pelo método DPPH e doseamento de flavonoides totais em extratos de folhas da Bauhinia variegata L. Rev Bras Farmácia. 2011;92(4):327-332.

36. Campos JS, Frasson APZ. Avaliação da atividade antioxidante do extrato aquoso de Lafoensia pacari A. ST.-Hil. em emulsão não-iônica. Rev Cienc Farm Básica Apl. 2011 Dec;32(3):363-8.

37. Oliveira DR, Brito-Junior FE, Bento EB, Matias EFF, Sousa ACA, Costa JGM, Coutinho HDM, Kerntopf MR, Menezes IRA. Antibacterial and modulatory effect of Stryphnodendron rotundifolium. Pharm Biol. 2011;49(12):1265-70, doi: 10.3109/13880209.2011.589857.

38. Costa JGM, Leite GO, Dubois AF, Seeger RL, Boligon AA, Athayde ML, Campos AR, Rocha JBT. Antioxidant effect of Stryphnodendron rotundifolium Martius extracts from Cariri-Ceará state (Brazil): Potential involvement in its therapeutic use. Molecules. 2012;17(1):934-50, doi: 10.3390/molecules17010934.

39. Zocoler AMD, Sanches ACC, Albrecht I, Mello JCP. Antioxidant capacity of extracts and isolated compounds from Stryphnodendron obovatum Benth. Braz J Pharm Sci. 2009 Sep;45(3):443-52, doi: 10.1590/S1984-82502009000300009.

40. Santos-Filho PR, Ferreira LA, Gouvêa CMCP. Protective action against chemical-induced genotoxicity and free radical scavenging activities of Stryphnodendron adstringens (" barbatimão") leaf extracts. Rev Bras Farmacogn. 2011 Sep;21(6):1000-5, doi: 10.1590/S0102-695X2011005000176.

41. Bona EAM, Pinto FGS, Fruet TK, Jorge TCM, Moura AC. Comparação de métodos para avaliação da atividade antimicrobiana e determinação da concentração inibitória mínima (CIM) de extratos vegetais aquosos e etanólicos. Arq Inst Biol. 2014 Sep;81(3):218-25, doi: 10.1590/1808-1657001192012.

42. Souza CMP, Brandão DO, Silva MSP, Palmeira AC, Simões MOS, Medeiros ACD. Utilização de plantas medicinais com atividade antimicrobiana por usuários do serviço público de saúde em Campina GrandeParaíba. Rev Bras Plantas Med. 2013;15(2):188-93, doi: 10.1590/S1516-05722013000200004.

43. Bessa NGF, Borges JCM, Beserra FP, Carvalho RHA, Pereira MAB, Fagundes R, Campos SL, Ribeiro LU, Quirino MS, Chagas Junior AF, Alves A. Prospecção fitoquímica preliminar de plantas nativas do cerrado de uso popular medicinal pela comunidade rural do assentamento vale verde - Tocantins. Rev Bras P1 Med. 2013;15(4):692-707, doi: 10.1590/S1516-05722013000500010.

44. Soares SP, Vinholis AHC, Casemiro LA, Silva MLA, Cunha WR, Martins CHG. Atividade antibacteriana do extrato hidroalcoólico bruto de Stryphnodendron adstringens sobre microorganismos da cárie dental. Rev Odonto Ciênc. 2008;23(2):141-4.

45. World Health Organization. Containing antimicrobial resistance. WHO Policy Perspectives on Medicines. v. 10. Geneva, Switzerland: WHO; 2005. 6 p.

46. Santos LS, Sant'Anna LO, Ramos JN, Ladeira EM, Stavracakis-Peixoto R, Borges LLG, Santos CS, Napoleão F, Camello TCF, Pereira GA, Hirata R, Vieira VV, Cosme LMSS, Sabbadini PS, MattosGuaraldi AL. Diphtheria outbreak in Maranhão, Brazil: microbiological, clinical and epidemiological aspects. Epidemiol Infect. 2015 May;143(4):791-8, doi: 10.1017/S0950268814001241.

47. Mina NV, Buurdz T, Wiebe D, Rai JS, Rahim T, Shing F, Hoang L, Bernard K. Canada's first case of a multidrug-resistant Corynebacterium diphtheriae strain, isolated from a skin abscess. J Clin Microbiol. 2011;49(11):4003-5, doi: 10.1128/JCM.05296-11.

48. Desoti VC, Maldaner CL, Carletto MS, Heinz AA, Coelho MS, Piati D, Tiuman TS. Triagem fitoquímica e avaliação das atividades antimicrobiana e citotóxica de plantas medicinais nativas da região oeste do estado do Paraná. Arq Cienc Saúde UNIPAR. 2011;15(1):3-13, doi: 10.25110/arqsaude.v15i1.2011.3686.

49. Stapper AP, Narasimhan G, Ohman DE, Barakat J, Hentzer SM, Molin S, Kharazmi A, Hoiby N, Mathee $\mathrm{K}$. Alginate production affects Pseudomonas aeruginosa biofilm development and architecture, but is not essential for biofilm formation. J Med Microbiol. 2004 Jul;53(7):679-90, doi: 10.1099/jmm.0.45539-0.

50. Díaz YM, Laverde GV, Gamba LR, Wandurraga HM, Arévalo-Ferro C, Rodríguez FR, Baltrán CD, Hernández LC. Biofilm inhibition activity of compounds isolated from two Eunicea species collected at the Caribbean Sea. Rev Bras Farmacogn. 2015;25(6):605-11, doi: 10.1016/j.bjp.2015.08.007. 
51. Noumi E, Snoussi M, Merghni A, Nazzaro F, Quindós G, Akdamar G, Mastouri M, Al-Sieni A, Ceylan. Phytochemical composition, anti-biofilm and anti-quorum sensing potential of fruit, stem and leaves of Salvadora persica L. methanolic extracts. Microbiol Pathog. 2017 Aug;109:169-76, doi: 10.1016/j.micpath.2017.05.036.

52. Sabbadini PS, Genovez MRN, Silva CF, Adelino TLM, Santos, CS, Pereira GA, Nagao PE, Dias AASO, Mattos-Guaraldi AL, Hirata Junior R. Fibrinogen binds to nontoxigenic and toxigenic Corynebacterium diphtheriae strains. Mem Inst Oswaldo Cruz. 2010 Aug;105(5):706-711, doi: 10.1590/s007402762010000500018.

53. Simpson-Louredo L, Ramos JN, Peixoto RS, Santos LS, Antunes CA, Ladeira EM, Santos CS, Vieira VV, Bôas MHSV, Hirata Junior R, Mattos-Guaraldi, AL. Corynebacterium ulcerans isolates from humans and dogs: fibrinogen, fibronectin and collagen-binding, antimicrobial and PFGE profiles. Antonie Van Leeuwenhoek. 2014 Feb;105(2):343-352, doi: 10.1007/s10482-013-0080-5

54. Ott L, Höller M, Rheinlaender J, Schäffer TE, Hensel M, Burkovski A. Strain-specific differences in pili formation and the interaction of Corynebacterium diphtheriae with host cells. BMC Microbiol. 2010 Oct;10:257, doi: 10.1186/1471-2180-10-257.

55. Peixoto RS, Antunes CA, Simpson-Lourêdo L, Viana VG, Santos CS, Silva JFR, Hirata Junior R, Hacker E, Mattos-Guaraldi AL, Burkovski A. Functional characterization of the collagen-binding protein DIP2093 and its influence on host-pathogen interaction and arthritogenic potential of Corynebacterium diphtheriae. Microbiol. 2017 May;163(5):692-701, doi: 10.1099/mic.0.000467.

56. Freitas ALD, Kaplum V, Rossi DCP, Silva LBR, Melhem MSC, Taborda CP, Mello JCP, Nakamura CV, Ishida K. Proanthocyanidin polymeric tannins from Stryphnodendron adstringens are effective against Candida spp. isolates and for vaginal candidiasis treatment. J Ethnopharmacol. 2018;216:184-90, doi: 10.1016/j.jep.2018.01.008.

57. Luiz RLF, Vila TVM, Mello JCP, Nakamura CV, Rozental S, Ishida K. Proanthocyanidins polymeric tannin from Stryphnodendron adstringens are active against Candida albicans biofilms. BMC Complement Altern Med. 2015 Mar;15:68, doi: 10.1186/s12906-015-0597-4.

58. Sper FL. Atividade antimicrobiana, anti-inflamatória, citotoxicidade e genotoxicidade do extrato glicólico de Stryphnodendron barbatiman (Vell.) Mart. (barbatimão) [dissertação]. São José dos Campos (SP): Universidade Estadual Paulista; 2018. $60 \mathrm{f}$.

59. Kumar MR, Aithal K, Rao BN, Udupa N, Rao BS. Cytotoxic, genotoxic and oxidative stress induced by 1,4-naphthoquinone in B16F1 melanoma tumor cells. Toxicol in Vitro. 2009 Mar;23(2):242-50, doi: 10.1016/j.tiv.2008.12.004.

60. Schiar VPP, Santos DB, Ludtke DS, Vargas F, Paixão MW, Nogueira CW, Zeni G, Rocha BT. Screening of potentially toxic chalcogens in erythrocytes. Toxicol in vitro. 2007;21:139-45, doi: 10.1016/j.tiv.2006.08.006.

61. Junqueira JC; Galleria mellonella as model host for human pathogens: recente studies and new perspective. Virulence. 2012;3:474-76, doi: 10.4161/viru.22493.

62. Baldivia DS, Leite DF, Castro DTH, Campos JF, Santos UP, Paredes-Gamero EJ, Carollo CA, Silva DB, Souza KP, Santos EL. Evaluation of in vitro antioxidant and anticancer properties of the aqueous extract from the stem bark of Stryphnodendron adstringens. Int J Mol Sci. 2018;19(8):2432, doi: 10.3390/ijms19082432.

63. Bukowska B, Kowalska S. Phenol and catechol induce prehemolytic and hemolytic changes in human erythrocytes. Toxicol Lett. 2004 Aug;152:73-84, doi: 10.1016/j.toxlet.2004.03.025.

64. War AR, Paulraj MG, Ahmad T, Buhroo AA, Hussain B, Ignacimuthu S, Sharma, HC. Mechanisms of plant defense against insect herbivores. Plant Signal Behav. 2012;7(10):1306-20, doi: 10.4161/psb.21663.

65. Cavalcante GM, Moreira AFC, Vasconcelos SD. Potencialidade inseticida de extratos aquosos de essências florestais sobre mosca-branca. Pesq Agropec Bras. 2006 Jan;41(1):9-14, doi: 10.1590/S0100204X2006000100002.

66. Isman MB. Botanical insecticides, deterrents, and repellents in modern agriculture and an increasingly regulated world. Annu Rev Entomol. 2006 Jan;51:45-66, doi: 10.1146/annurev.ento.51.110104.151146.

67. Inoue MH, Santana DC, Pereira MJB, Possamai ACS, Azevedo VH. Extratos aquosos de Xylopia aromatica e Annona crassiflora sobre capim-marandu (Brachiaria brizantha) e soja. Sci Agrac. 2009; 10(3):245-50.

68. Ribeiro LO, Barbosa S, Baleiro FP, Beijo LA, Santos BR, Gouvea CMCP, Paiva LV. Fitotoxicidade de extratos foliares de barbatimão [Stryphnodendron adstringens (Mart.) Coville] em bioensaio com alface. Rev Bras Bioc. 2012 Jun;10(2):220-5.

69. Formagio ASN, Masette TE, Trevizan LNF, Vidoieira MC. Potencial alelopático de Stryphnodendron adstringens (Mart) Coville na germinação e crescimento inicial de picão-preto. Iheringia Série Botânica. 2018 May;73(1):60-4, doi: 10.21826/2446-8231201873108. 